\title{
A train of blue light pulses delivered through closed eyelids suppresses melatonin and phase shifts the human circadian system
}

This article was published in the following Dove Press journal:

Nature and Science of Sleep

3 October 2013

Number of times this article has been viewed

\section{Mariana G Figueiro \\ Andrew Bierman \\ Mark S Rea}

Lighting Research Center, Rensselaer Polytechnic Institute, Troy, NY, USA
Correspondence: Mariana G Figueiro Lighting Research Center,

Rensselaer Polytechnic Institute,

21 Union Street, Troy, NY 12180, USA

$\mathrm{Tel}+\mathrm{I} 5186877100$

$\mathrm{Fax}+\mid 5186877120$

Email figuem@rpi.edu
Abstract: A model of circadian phototransduction was published in 2005 to predict the spectral sensitivity of the human circadian system to narrow-band and polychromatic light sources by combining responses to light from the spectral-opponent "blue" versus "yellow" cone bipolar pathway with direct responses to light by the intrinsically photosensitive retinal ganglion cells. In the model, depolarizing "blue" responses, but not hyperpolarizing "yellow" responses, from the "blue" versus "yellow" pathway are combined with the intrinsically photosensitive retinal ganglion cell responses. Intrinsically photosensitive retinal ganglion cell neurons are known to be much slower to respond to light than the cone pathway, so an implication of the model is that periodic flashes of "blue" light, but not "yellow" light, would be effective for stimulating the circadian system. A within-subjects study was designed to test the implications of the model regarding retinal exposures to brief flashes of light. The study was also aimed at broadening the foundation for clinical treatment of circadian sleep disorders by delivering flashing light through closed eyelids while people were asleep. In addition to a dark control night, the eyelids of 16 subjects were exposed to three light-stimulus conditions in the phase delay portion of the phase response curve while they were asleep: (1) 2 -second flashes of $111 \mathrm{~W} / \mathrm{m}^{2}$ of blue $\left(\lambda_{\max }\right.$ $\approx 480 \mathrm{~nm}$ ) light once every minute for 1 hour, (2) $131 \mathrm{~W} / \mathrm{m}^{2}$ of green $\left(\lambda_{\max } \approx 527 \mathrm{~nm}\right)$ light, continuously on for 1 hour, and (3) 2-second flashes of the same green light once every minute for 1 hour. Inferential statistics showed that the blue flash light-stimulus condition significantly delayed circadian phase and significantly suppressed nocturnal melatonin. The results of this study further our basic understanding of circadian phototransduction and broaden the technical foundations for delivering light through closed eyelids during sleep for treating circadian sleep disorders.

Keywords: melatonin, dim light melatonin onset, eyelids, flashing blue light, circadian rhythms, sleep

\section{Introduction}

Recently, Zeitzer et al reported that a sequence of 2-millisecond light pulses presented every minute for 60 minutes to six subjects with eyes opened produced an average phase shift of approximately 45 minutes, as measured by dim light melatonin onset (DLMO), a marker of the circadian clock, and improved objective (electroencephalogram) and subjective (Stanford Sleepiness Scale) alertness. ${ }^{1}$ While there was a reduction in melatonin concentrations with the 2-millisecond light pulses, the authors reported a nonsignificant difference in melatonin concentrations between the 2-millisecond light pulse and the dark control night. Similar phase shift findings for pulsed light have been demonstrated using animals. ${ }^{2,3}$ 
On the surface, these findings might appear at odds with conventional conceptualizations of the phototransduction properties of the human circadian system and with models of the response to light by the human circadian pacemaker., ${ }^{4,5}$ A number of studies have shown that melatonin suppression and phase shifting have similar spectral and absolute sensitivities to light. ${ }^{6-8}$ Similarly, prior to the recent publication from Zeitzer et al, ${ }^{1}$ most studies have shown, usually through melatonin suppression alone, that a prolonged light stimulus (ie, longer than several milliseconds) will reliably stimulate the human circadian system. ${ }^{69}$ Figueiro et $\mathrm{al}^{9}$ showed that the exposure duration needed to suppress melatonin is dependent upon the magnitude of the light stimulus. For example, they reported that continuous exposure to $74 \mu \mathrm{W} / \mathrm{cm}^{2}$ of a narrow-band, short wavelength light stimulus (peak close to $470 \mathrm{~nm}$ ) resulted in measureable melatonin suppression after 5-10 minutes, and continuous exposure to $2 \mu \mathrm{W} / \mathrm{cm}^{2}$ of the same blue light for 90 minutes was needed to measurably suppress melatonin. ${ }^{9}$ Chang et $\mathrm{al}^{6}$ demonstrated that a 12-minute exposure to $4100 \mathrm{~K}$ fluorescent light $(>6000 \mathrm{~lx}$ at the cornea) was more effective in phase shifting circadian rhythms than longer durations (eg, 6.4 hours).

Although the underlying retinal mechanisms responsible for photic stimulation of the circadian system are still under investigation, Rea et al, in two separate reports, have proposed a model of human circadian phototransduction. ${ }^{10,11}$ The proposed model, consistent with known retinal neuroanatomy and neurophysiology, was shown to be able to quantitatively predict light-induced nocturnal melatonin suppression from both narrow-band and polychromatic light sources..$^{9,12,13}$ All of these studies of melatonin suppression utilized prolonged light stimuli to human subjects, so the impact of temporally modulated light stimuli remained unexplored. Nevertheless, as discussed below, qualitative predictions of the impact of temporally modulated light pulses for photic stimulation of the human circadian system are possible from the model.

The intrinsically photosensitive retinal ganglion cell (ipRGC) $)^{14}$ is a central element in the phototransduction model, and it has been demonstrated that indirect input from rods and cones also affects the photic information passed to the master pacemaker located in the suprachiasmatic nuclei (SCN). Soon after discovery of the ipRGCs, a series of studies using animal models showed that circadian phase shifting and entrainment can occur by stimulation of the ipRGCs alone, by rods and cones alone, and of course, with all photoreceptors providing input in combination. ${ }^{15,16}$ According to the model by Rea et al, ${ }^{10}$ photic information from cones is communicated to the SCN via a complex of synapses in the retina.
More specifically, spectrally opponent (blue versus yellow) S-cone bipolar neurons combine input from all three cone types that, in turn, provide a depolarizing-only (S-ON) input to the ipRGCs. Axons from these ipRGCs form the retinohypothalamic tract and carry photic information to the SCN. According to the phototransduction model, a depolarizing "blue" response from the S-ON bipolar neurons can add to the intrinsically photosensitive response from the ipRGC, but a hyperpolarizing "yellow" response cannot. For light spectra that evoke a "yellow" response from the S-ON bipolar, photic information to the $\mathrm{SCN}$ is determined by the response of the ipRGC alone.

It is well known that cones respond very quickly to a light stimulus ( $<50$ milliseconds) ${ }^{17}$ and that the ipRGC is slower (>10 seconds) to respond. ${ }^{14}$ Moreover, cones will exhibit a rapid, heightened response to the onset of a bright pulse of light, followed by a slow decay, even after the stimulus is extinguished..$^{18}$ Hypothetically, bright, short wavelength light flashes could stimulate the SCN via the depolarizing, S-ON bipolar synapse with the ipRGC, without necessarily evoking a direct, high-threshold and slow-responding phototransducing response from these ipRGCs. Therefore, the model is consistent with the hypothesis that a train of brief short wavelength flashes of light that predominately stimulate S-cones (a "blue" response) could provide photic information to the SCN. Conversely, a train of brief longer-wavelength green, yellow, or red flashes of light (a "yellow" response) would not surpass the high threshold and slow responding ipRGC sensitivity.

The spectral transmittance of the human eyelid has recently been determined by Bierman et al. ${ }^{19}$ The efficacy of a sleep mask delivering 60 minutes of continuous 527-nm (green) light for stimulating the human circadian system through the eyelids while subjects were asleep has also been demonstrated, both in terms of nocturnal melatonin suppression and of phase delaying DLMO.${ }^{13}$ This method of delivering light while people sleep offers great promise for clinical studies aimed at correcting circadian misalignment because light applied close to the minimum core body temperature $\left(\mathrm{CBT}_{\text {min }}\right)$ will maximally shift the timing of the circadian pacemaker; ${ }^{20,21} \mathrm{CBT}_{\text {min }}$ occurs in the second half of the night, approximately 2 hours prior to natural waking.

The present study was designed to further the basic understanding of circadian phototransduction by exploring the theoretical hypothesis that brief pulses of short wavelength light (blue) could evoke a circadian phase shift and nocturnal melatonin suppression, while brief pulses of longer-wavelength (green) light would not evoke the same 
response by the circadian system. A secondary goal of the present study was to broaden the technical foundations for future clinical applications of light delivery through the closed eyelids of sleeping patients.

\section{Materials and methods Subject selection}

Sixteen subjects (five females), mean age \pm standard deviation (SD) $40 \pm 10.7$ years were recruited to participate in the study from an electronic posting at Rensselaer Polytechnic Institute in Troy, NY. All subjects were screened for major health problems and reported not taking any pharmaceuticals or medications. All subjects provided written informed consent approved by the Institute Review Board at Rensselaer Polytechnic Institute and were paid for their participation. Subjects were asked to refrain from alcohol and caffeine starting 12 hours prior to coming to the lab on the days of the experiment.

Every subject completed a Munich Chronotype Questionnaire (MCTQ) prior to the study. ${ }^{22}$ Average \pm SD chronotype was $2.1 \pm 1.6$. Participants completed a sleep/ wake diary during all weeks of the experiment, beginning 1 week prior to the start of the experiment. These diaries documented bedtimes, rising times, caffeine consumption, and quality of sleep. All subjects were asked to maintain a regular sleep/wake cycle (bedtime at $11 \mathrm{pm}$ and wake time at $7 \mathrm{am}$ ) starting 2 weeks prior to and during the 4 weeks of the experiment. Sleep logs were verified with a Dimesimeter ${ }^{23}$ worn on the nondominant wrist of each subject. The sleep schedule subjects were required to maintain during the experimental weeks was within 60-90 minutes from their habitual bed/wake times. Subjects were not allowed to deviate from required sleep schedules by more than \pm 30 minutes.

\section{Lighting conditions}

Light was delivered to both eyes through the closed eyelids of subjects using the light mask previously described by Figueiro and Rea. ${ }^{13}$ Every light mask contained two light emitting diode (LED) arrays, one for each eye, that were held in position in front of the eyelids by an elastic strap around the back of the head. Three light-stimulus conditions (in addition to a dark control condition) were used: (1) 2-second flashes of $111 \mathrm{~W} / \mathrm{m}^{2}$ blue light (wavelength of peak intensity $\left[\lambda_{\max }\right]=480 \mathrm{~nm}$, full width half maximum $\left.[\mathrm{FWHM}]=24 \mathrm{~nm}\right)$ presented once every minute for 60 minutes (flashing blue light), (2) $131 \mathrm{~W} / \mathrm{m}^{2}$ of green light $\left(\lambda_{\max }=527 \mathrm{~nm}\right.$, $\mathrm{FWHM}=33 \mathrm{~nm}$ ), continuously on for 60 minutes (continuous green light), and (3) 2-second flashes of the $131 \mathrm{~W} / \mathrm{m}^{2}$ green $\operatorname{light}\left(\lambda_{\max }=527 \mathrm{~nm}, \mathrm{FWHM}=33 \mathrm{~nm}\right)$ presented once every minute for 60 minutes (flashing green light).

The mean eyelid spectral transmittance function from Bierman et $\mathrm{al}^{19}$ was used to determine corneal irradiance for a closed eyelid, where the mean transmittance for the blue light is 0.0028 and 0.0053 for the green light. Light mask corneal irradiance levels were chosen based on the calculated circadian stimulus (CS) value for the continuous green light stimulus. ${ }^{24} \mathrm{CS}$ is equivalent to the estimated percentage of light-induced nocturnal melatonin suppression following 1 hour exposure to the retina (eyes open) from a non-flashing light through a fixed pupil of $2.3 \mathrm{~mm}$ diameter. The CS values for the blue and green lights were 0.31 and 0.38 , respectively. Tables 1 and 2 provide quantitative estimates of the light stimuli presented to the subjects in a counterbalanced manner.

There are three categories of uncertainty in the photometric values in Table 1. First, there is uncertainty in the photometric measurements. Combining the sources of error for measurement of the light mask, the uncertainty associated with any given value is approximately $7 \%$ for the particular methods used when these measurements were obtained in the Lighting Research Center laboratory, a National Voluntary Laboratory Accreditation Program (NVLAP) accredited photometric laboratory (NVLAP lab code: 200480-0). Greater uncertainty is associated with the measurement of brief light pulses, so our estimated photometric uncertainty for the flashing blue and green lights stimuli is approximately $10 \%$. The second category of uncertainty is associated with setting the light output from the 527-nm LEDs for the conditions of the experiment. The light mask irradiances for the 480-nm LED arrays were adjusted precisely using pulse-width modulation so that the measured values for the left and the right arrays ranged from 110-111 W/m ${ }^{2}$ (mean $\pm \mathrm{SD}, 111 \mathrm{~W} / \mathrm{m}^{2} \pm 1 \%$ ). The 527-nm LEDs, on the other hand, had to be operated near their maximum light

Table I Light mask irradiance and illuminance

\begin{tabular}{|c|c|c|c|c|}
\hline Quantity & Units & $\begin{array}{l}\text { Blue flash } \\
(480 \mathrm{~nm})\end{array}$ & $\begin{array}{l}\text { Green flash } \\
(527 \mathrm{~nm})\end{array}$ & $\begin{array}{l}\text { Green continuous } \\
(527 \mathrm{~nm})\end{array}$ \\
\hline Irradiance on eyelid & $\mathrm{W} / \mathrm{m}^{2}$ & 111 & 131 & 131 \\
\hline Illuminance on eyelid & lux & 16,400 & 74,900 & 74,900 \\
\hline
\end{tabular}


Table 2 Light mask stimulus quantities

\begin{tabular}{|c|c|c|c|c|}
\hline Quantity & Units & $\begin{array}{l}\text { Blue flash } \\
(480 \mathrm{~nm})\end{array}$ & $\begin{array}{l}\text { Green flash } \\
(527 \mathrm{~nm})\end{array}$ & $\begin{array}{l}\text { Green continuous } \\
(527 \mathrm{~nm})\end{array}$ \\
\hline $\begin{array}{l}\text { Mean eyelid optical } \\
\text { density } \pm S D \text { (transmittance) }{ }^{19}\end{array}$ & NA & $\begin{array}{l}2.56 \pm 0.31 \\
(0.0028)\end{array}$ & $\begin{array}{l}2.27 \pm 0.26 \\
(0.0053)\end{array}$ & $\begin{array}{l}2.27 \pm 0.26 \\
(0.0053)\end{array}$ \\
\hline Corneal irradiance & $\mathrm{W} / \mathrm{m}^{2}$ & 0.31 & 0.70 & 0.70 \\
\hline Corneal illuminance* & lux & 59 & 380 & 380 \\
\hline $\mathrm{CL}_{\mathrm{A}}$ & NA & 290 & 410 & 410 \\
\hline CS & NA & 0.31 & 0.38 & 0.38 \\
\hline Integrated stimulus & W second $/ \mathrm{m}^{2}$ & 37 & 84 & 2,500 \\
\hline (I hour) on cornea & lux second & $7.1 \times 10^{3}$ & $4.5 \times 10^{4}$ & $1.4 \times 10^{6}$ \\
\hline
\end{tabular}

Note: *based upon luminous transmittance of eyelids.

Abbreviations: $C S$, circadian stimulus; $N A$, not applicable; $S D$, standard deviation; $\mathrm{CL}_{\mathrm{A}}$, Circadian illuminance.

output for the conditions of the experiment, so it was not possible to make fine adjustments of their light output using pulse-width modulation. Consequently, the measured light mask irradiance values for the left and right arrays ranged from $104-147 \mathrm{~W} / \mathrm{m}^{2}$ for the 527-nm LEDs (mean $\pm \mathrm{SD}$, $131 \mathrm{~W} / \mathrm{m}^{2} \pm 14 \%$ ). The third category of stimulus uncertainty arises from anatomical differences among subjects and the exact positioning of the light mask on the face of each subject. Laboratory measurements of the light masks were taken at a particular distance $(39 \mathrm{~mm})$ and location in front of the mask corresponding to an estimate of the location of a wearer's eyelid. Based on irradiance measurements when moving the detector within the range of expected eyelid locations, the variation of eyelid irradiance levels was approximately $2 \%$ per millimeter in any direction.

\section{Procedures}

All subjects arrived at the lab at $6.30 \mathrm{pm}$ on the first evening (E1) of an experimental session and completed the session approximately 30 hours later (midnight on the second evening [E2] of an experimental session). Each subject participated in four 30-hour experimental sessions, 2 weeks apart. DLMO phase was assessed with saliva samples collected every 20 minutes, starting at $7 \mathrm{pm}$ and ending at $11.20 \mathrm{pm}$ on E1 and starting at $7 \mathrm{pm}$ until midnight on E2. Figure 1 illustrates the protocol that was repeated for four consecutive weekends. Saliva was collected using the Salivette system (Sarstedt AG and Co, Nümbrecht, Germany). Saliva samples were frozen at $-20^{\circ} \mathrm{C}$ until assayed for melatonin levels. All saliva samples from one experimental night were assayed in the same batch. To prevent contamination of the saliva samples, subjects were not allowed to eat or drink between sample times.

Following the last saliva sample collection on E1 and before subjects were allowed to sleep, a registered nurse inserted an indwelling catheter into the vein of each subject to allow blood sample collection during sleep. Blood samples were used to assess plasma melatonin concentrations prior to

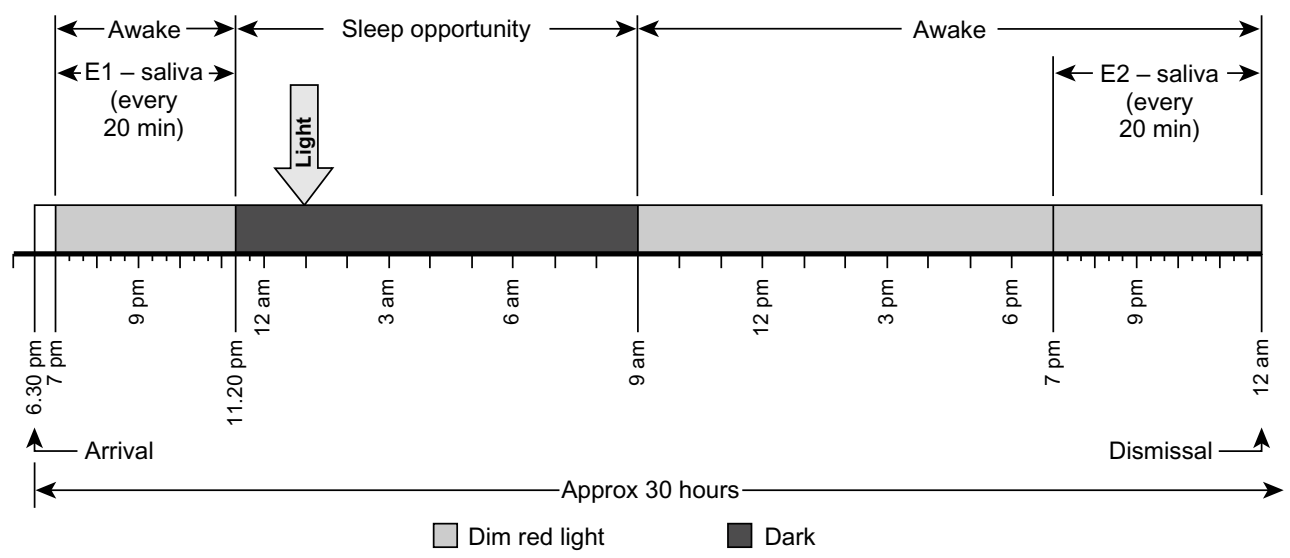

Figure I Experimental protocol for each 30-hour session.

Notes: Each of four 30 hour sessions began at 6.30 pm. Subjects were given a sleep opportunity at II.30 pm; otherwise they were awake in dim red light (<I Ix at cornea). Saliva samples for DLMO calculations were taken during both evenings (EI and E2) of each session every 20 minutes, starting both evenings at 7 pm and ending at II.20 pm during the first evening (EI) and midnight during the second evening (E2). Each session was associated with a single lighting condition: dark, continuous green light, flashing green light, and flashing blue light. All lighting conditions, including dark, were presented 90-120 minutes after the sleep opportunity period began (large arrow). One blood sample was collected immediately prior to light exposure and another blood sample was collected immediately after.

Abbreviation: DLMO, dim light melatonin onset. 
and just after exposure to each lighting condition. Subjects were on their mattresses by $11.30 \mathrm{pm}$ and were allowed to sleep until 9.00 am the following day. Except for the dark control night condition, the light mask was energized and delivered each of the lighting conditions described above at approximately 90-120 minutes after subjects were allowed to sleep. The light mask was energized approximately 2 hours prior to each subject's predicted $\mathrm{CBT}_{\min }$. The predicted $\mathrm{CBT}_{\text {min }}$ was determined using each subject's sleep-log times $^{25}$ obtained prior to the experiment, and the range for the 16 subjects was between 3 am and $4.30 \mathrm{am}$. The light exposures occurred between 1 am and $2.30 \mathrm{am}$; therefore, the light stimuli were scheduled to be presented while subjects were asleep and prior to their $\mathrm{CBT}_{\min }$, that is, during the expected delay portion of the phase response curve (PRC). Consequently, DLMO during E2 should have been delayed relative to that during $\mathrm{E} 1$ and, because of the light-condition stimulus, delayed relative to that recorded during E1 during the dark control night. In order to ensure that the subjects' DLMO did not vary significantly from week to week, and therefore that the light exposures for all experimental conditions were given at similar circadian times. DLMO times obtained during E1 for all 4 weeks of the experiment were statistically compared using two-tailed paired Student's $t$-tests. There were no significant differences in DLMO times between weeks $(P>0.05)$. Parenthetically, post hoc analyses of predicted $\mathrm{CBT}_{\min }$, calculated using the measured DLMO times obtained in E1 plus 7 hours, showed that the predicted range of $\mathrm{CBT}_{\text {min }}$ was between $2.40 \mathrm{am}$ and $6.00 \mathrm{am}$.

In order to investigate the impact of each lighting condition on nocturnal melatonin suppression levels, one blood sample was drawn from the indwelling catheter immediately prior to light exposure, and another was drawn immediately after. After all blood samples were collected, the indwelling catheter was removed and participants were allowed to remove the light mask and return to sleep until 9 am that morning.

From 9 am until $7 \mathrm{pm}$ on E2, subjects remained in dim red $\left(\lambda_{\max }=630 \mathrm{~nm}, \mathrm{FWHM}=22 \mathrm{~nm}\right) \operatorname{light}(<1 \mathrm{~lx}$ at the cornea $)$ and were allowed to perform normal computer work, read, and play games. Subjects remained sitting at all times, except for using the restrooms, which were also illuminated with low levels of red light. Electronic devices (ie, computers, phones, portable media players) were dimmed to the lowest possible brightness and were covered with an orange filter (Roscolux \# 21 Golden Amber; Rosco, Stamford, CT, USA); spectral transmittance of these orange filters was not greater than $2 \%$, from $380-550 \mathrm{~nm}$. Napping was not allowed during the experiment. Breakfast, lunch, and dinner were offered at specific times. Subjects were asked to brush their teeth after dinner, which was offered 60 minutes prior to data collection for the second DLMO assessment on E2. DLMO phase was assessed again with saliva samples collected every $20 \mathrm{~min}$, starting at $7 \mathrm{pm}$ and ending at midnight on E2, after which subjects were allowed to go home.

\section{Data analyses \\ Phase shifting}

DLMO thresholds for each melatonin profile from subjects were calculated using two techniques published in the literature by taking the average of the three lowest points plus twice the standard deviation of these points (" $3 \mathrm{~L}$ ") ${ }^{26}$ and by taking the average of the five continuous lowest points plus $15 \%$ of the five continuous highest points (" $5 \mathrm{H} / 5 \mathrm{~L}$ ") ${ }^{27}$. The two different DLMO threshold calculations were employed to account for the different melatonin profiles in the subjects, as shown by visual inspection of the data. ${ }^{28}$ Subjects whose DLMO thresholds were calculated using the $3 \mathrm{~L}$ method had steeper melatonin profiles, while subjects whose DLMO thresholds were calculated using the $5 \mathrm{H} / 5 \mathrm{~L}$ method exhibited less steep melatonin profiles at the start of data collection until later in the evening, when melatonin levels went up; therefore, the $5 \mathrm{H} / 5 \mathrm{~L}$ threshold, which included the higher levels at the end of the night in the calculation, better characterized DLMO thresholds for these subjects because the first three lowest points were very often the same value. The DLMO time for each profile was the time (as determined by linear interpolation) that the fitted curve exceeded and remained above the DLMO threshold. Phase shifting was determined by subtracting DLMO obtained during the second evening from DLMO obtained during the first evening. A negative difference means the subject had a delayed DLMO on the second evening relative to the first evening, 24 hours earlier. Two-tailed, one-sample $t$-tests were performed to determine if phase shifting obtained under each lighting condition was statistically different than zero. In order to determine whether phase shift was significantly different after exposure to each lighting condition compared to the dark control condition, two-tailed, paired $t$-tests were performed comparing the phase shift obtained during the dark control condition and all three lighting conditions. Bonferroni corrections were applied. PASW Statistics 18.0 software was used (IBM Corporation, Armonk, NY, USA).

In order to account for the natural drift of the circadian clock that occurs when subjects remain in dim light, the phase shift exhibited in the dark (DLMO E1-DLMO E2), control lighting condition was subtracted from those obtained in 
each of the three lighting conditions, when the light mask was energized (ie, an adjusted phase shift was calculated). For each subject, the phase shift obtained in the dark control condition was subtracted from those obtained in the flashing blue, continuous green and flashing green lighting conditions (eg, [DLMO E1-DLMO E2 $]_{\text {flashing blue }}-$ [DLMO E1-DLMO E2 $]_{\text {dark }}$ ). Two-tailed, one-sample $t$-tests were performed to determine if the adjusted phase shifts obtained under each lighting condition (flashing blue, continuous green, flashing green) were statistically different than zero.

\section{Acute melatonin suppression}

Adjusted melatonin suppressions were calculated using adjusted dark values for each subject to account for their natural rise in melatonin level concentrations while in the dark and for week-to-week differences in the initial melatonin concentrations. Melatonin concentrations obtained from each subject during the initial dark condition were used for the adjusted dark values. Melatonin suppression was calculated for each lighting condition using the adjusted dark value at the same sampling time. The adjusted dark value for a given lighting condition and sampling time is given as,

$$
\mathrm{A}=\left(\mathrm{C}_{\mathrm{T} 1, \mathrm{Lm}} / \mathrm{C}_{\mathrm{T} 1, \mathrm{D}}\right) \mathrm{C}_{\mathrm{T} 2, \mathrm{D}}
$$

where $\mathrm{A}$ is adjusted dark values, $\mathrm{C}$ is melatonin concentration $(\mathrm{pg} / \mathrm{mL})$; $\mathrm{T}$ is sampling time $(\mathrm{n}=1$ or $2 ; 1$ is prior to energizing the mask and 2 is 60 minutes after energizing the mask); L is lighting condition per experimental session ( $\mathrm{m}=1,2$, or $3 ; 1$ is flashing blue, 2 is continuous green, and 3 is flashing green), D is dark conditions. Melatonin suppression $\mathrm{S}$ is,

$$
\mathrm{S}=100 *\left(1-\left[\mathrm{C}_{\mathrm{T} 2, \mathrm{Lm}} / \mathrm{A}\right]\right)
$$

\section{Results}

\section{Phase shifting}

Figure 2 shows the phase shift obtained in each experimental condition for every subject $(n=16)$ as well as the mean \pm standard error of the mean (SEM) phase shifts (in minutes); tabulated values are given in Table 3. One-sample, two-tailed $t$-tests revealed that phase shifts obtained in the flashing blue, the continuous green, and the flashing green lighting conditions were statistically different than zero $(P=0.001$, $P=0.012$, and $P=0.006$, respectively). Phase shifts after the dark control condition were not reliably different than zero $(P=0.1)$. Two-tailed, paired $t$-tests revealed a significant

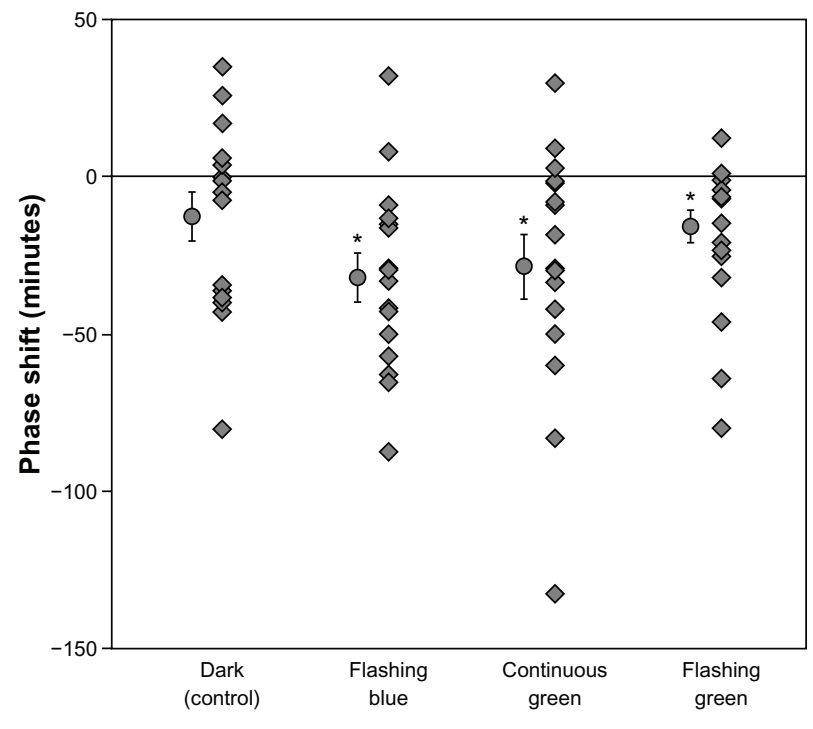

Figure 2 Individual phase shifts in minutes (ordinate) for 16 subjects who completed the four 30-hour experimental sessions (filled diamonds).

Notes: A negative value means that dim light melatonin onset (DLMO) on the second evening occurred later than DLMO on the first evening. The mean \pm standard error of the mean are also shown (filled circles). *Represents phase shifts significantly greater than zero $(P<0.05)$. When DLMO phase shift was adjusted to account for the natural drift that occurs in the dark (control) night, phase shift was significantly greater than zero $(P<0.05)$ only after exposure to flashing blue light. Abbreviation: DLMO, dim light melatonin onset.

difference between the dark control condition and the flashing blue lighting condition $(P=0.05)$, but the differences between the dark control condition and the continuous green lighting condition and the flashing green lighting conditions were not statistically significant $(P=0.2$ and $P=0.5$, respectively). Since, on average, the human circadian clock has a period slightly longer than 24 hours, the lack of morning light exposure would be expected to delay the clock. However, about $10 \%$ of the population has a circadian clock with a period slightly shorter than 24 hours, ${ }^{29}$ and darkness during the day would be expected to exhibit a phase advance in DLMO the subsequent evening for these individuals. In the present study, 5 subjects (31\%) exhibited a slight phase advance of their DLMO after remaining in darkness.

Subject-specific adjusted phase shifts for each lighting condition relative to the dark condition were calculated. Onesample, two-tailed $t$-tests revealed that the flashing blue light condition was significantly different than zero $(P=0.05)$. The mean \pm SEM and median adjusted phase shifts for the three lighting conditions are shown in Table 3.

\section{Acute melatonin suppression}

Figure 3 shows the adjusted melatonin suppression for the three lighting conditions; tabulated values are given in Table 3. Two-tailed, one-sample $t$-tests revealed that suppression was significantly greater than zero after the 
Table 3 Phase shift, adjusted phase shifts, and nocturnal melatonin suppression for the four 30-hour experimental sessions

\begin{tabular}{lllll}
\hline & Dark & $\begin{array}{l}\text { Flashing } \\
\text { blue }\end{array}$ & $\begin{array}{l}\text { Continuous } \\
\text { green }\end{array}$ & $\begin{array}{l}\text { Flashing } \\
\text { Green }\end{array}$ \\
\hline $\begin{array}{l}\text { Phase shift (min) } \\
\text { Mean } \pm \text { SEM }\end{array}$ & $-12 \pm 3$ & $-32 \pm 8^{*}$ & $-29 \pm 10^{*}$ & $-20 \pm 6^{*}$ \\
$\quad \begin{array}{l}\text { Median } \\
\text { Adjusted phase }\end{array}$ & -3 & -31 & -24 & -11 \\
shift (min) & & & \\
$\quad \begin{array}{l}\text { Mean } \pm \text { SEM } \\
\text { Median }\end{array}$ & $-20 \pm 9 *$ & $-16 \pm 13$ & $-8 \pm 11$ \\
$\begin{array}{l}\text { Melatonin } \\
\text { suppression }\end{array}$ & -22 & -13 & -4 \\
$\quad$ & & & \\
$\quad$ Mean \pm SEM & & $14 \% \pm 5^{*}$ & $56 \% \pm 4^{* *}$ & $7 \% \pm 5$ \\
\hline
\end{tabular}

Notes: The mean \pm standard error of the mean (SEM) and the median values are shown. The adjusted phase shifts (in minutes) accounted for the natural drift in circadian phase that occurred when subjects remained in the dark; *P $<0.05 ; * * P<0.01$.

continuous green $(P<0.0001)$ and after the blue flash $(P=0.02)$ light stimuli, but not after the green flash light stimulus $(P=0.26)$.

\section{Discussion}

The present study was designed to determine whether a train of blue light pulses and of green light pulses ( 2 seconds on, 58 seconds off, for 60 minutes) delivered through closed eyelids could, while subjects slept, suppress nocturnal melatonin and delay DLMO. After correcting for mean eyelid transmittance, the corneal irradiance levels of the flashing blue and both green light conditions were set to values previously shown to be of approximately equal effectiveness for stimulating the human circadian system for a continuous exposure of 1 hour ( $\mathrm{CS}=0.31$ for blue and 0.38 for green). Results showed that, when corrected for the natural drift of

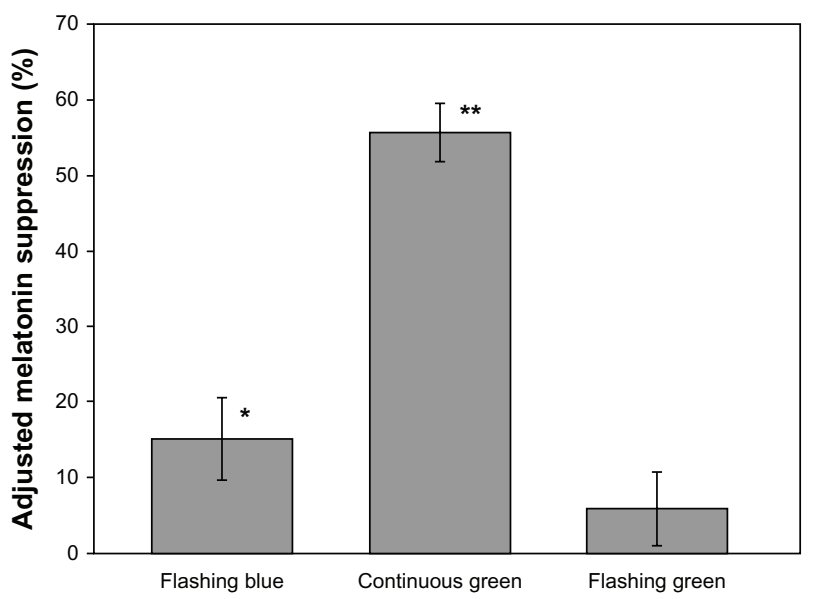

Figure 3 Mean \pm standard error of the mean adjusted melatonin suppression for the three lighting conditions.

Note: Melatonin suppression significantly greater than zero $(* P<0.05$ or $* * P<0.01)$. circadian phase, the flashing blue lighting condition reliably induced a phase delay as measured by the incremental change in DLMO relative to the dark control condition. Circadian phase following the flashing green lighting condition was not, however, reliably different than the natural drift in circadian phase in the dark. The flashing blue lighting condition also reliably suppressed nocturnal melatonin, but the flashing green lighting condition did not.

These results are consistent with our hypothesis that the train of blue light pulses, but not the train of green light pulses, could reliably affect phase shifting and melatonin suppression. This suggests that, as predicted by the model proposed by Rea et al, ${ }^{10,11}$ short wavelength, narrow-band stimuli $\left(\lambda_{\text {peak }}<507 \mathrm{~nm}\right)$ have different temporal signaling characteristics than longer wavelength stimuli matched for equal effectiveness when presented continuously. According to the model, the S-ON bipolar can communicate depolarizing "blue" information but not hyperpolarizing "yellow" information to the ipRGCs. ${ }^{10}$ Thus, any photic information from long wavelength light (longer than approximately $507 \mathrm{~nm}$ ) communicated to the SCN is posited to be the result of the intrinsic photosensitive nature of the ipRGCs. Given the relatively fast temporal dynamics of cones and the sluggish response to light by the ipRGCs, ${ }^{14}$ it was hypothesized that the flashing blue lighting condition could convey photic information to the $\mathrm{SCN}$ via the ipRGC without necessarily evoking a direct, phototransducing response to light by the ipRGC. In contrast, the flashing green lighting condition, because it evokes a hyperpolarizing "yellow" response in the S-ON bipolar, simply cannot communicate photic information to the ipRGCs. Moreover, the ipRGC would not be able to evoke a significant direct, phototransducing response to the train of green light pulses because their total energy level (irradiance $\times$ time) was a factor of 30 less than that of a continuous green light of the same irradiance. Indeed, and as previously shown, ${ }^{13}$ the continuous green light of the same corneal irradiance delivered through the closed eyelid in the present study was able to reliably suppress melatonin and, although not statistically significant, delay circadian phase relative to darkness.

The absolute phase shifting amounts obtained in the present study were lower than those obtained by Zeitzer et al. ${ }^{1}$ They reported using "white" light flashes of 2-millisecond duration, repeated once every 60 seconds for 1 hour at a level of 473 lx. A plot of the spectral power distribution of their white light source is provided in their paper. Their plot indicates that a xenon discharge lamp was used, presumably a xenon flash lamp, to deliver the light stimulus. Xenon flash 
lamps, by their nature deliver extremely intense and extremely short pulses of light. ${ }^{30}$ Based on the light measurement equipment specified in their paper, it can only be assumed that the reported illuminance was a time-averaged photometric value recorded over a period of seconds and not an instantaneous value during the flash. Although unspecified, the actual corneal illuminance of the xenon flash must have been much higher than 473 1x. This inferred difference between their reported and the expected stimulus magnitude may explain the relatively larger phase shift in their study compared to the present one. Specifically, bleached retinal photopigments from a xenon flash will continue to signal "light" to the brain well after the light has been extinguished.

Another possible explanation for the smaller phase shift in the present study may be that the light stimuli may not have been restricted to the delay portion of the PRC or may have been delivered farther from the $\mathrm{CBT}_{\text {min }}$ than originally predicted using sleep logs. Most of the subjects in the present study exhibited DLMO on the first evening that occurred 3-5 hours prior to the start of the light exposures. Jewett et $\mathrm{al}^{20}$ and Khalsa et $\mathrm{al}^{21}$ proposed a transition from delaying effects of light to advancing effects of light in the PRC (crossover point) at the time of $\mathrm{CBT}_{\min }$ (estimated to occur about 7 hours after DLMO). However, precise estimation of $\mathrm{CBT}_{\text {min }}$ is difficult in the field and the phase angle between DLMO and $\mathrm{CBT}_{\text {min }}$ may vary among individuals; therefore, the duration of light exposures in the present study may have extended into the phase advance portion of the PRC for some subjects, thereby reducing the magnitude of the average phase delay. This possibility may also explain the apparent disparity between the rather modest average phase delay and the very high average level of nocturnal melatonin suppression resulting from exposure to the continuous green light stimulus. In other words, the net circadian phase change will depend upon light exposures in both the advance and delay portions of the PRC, but the amount of melatonin suppressed does not. It is recommended that a similar study be performed while light is applied at an earlier time (eg, soon after subjects fall asleep).

It should also be noted that the continuous green light evoked a much higher level of melatonin suppression than would be expected from the CS calculation. Since the same eyelid irradiance levels were used for all subjects, the high suppression levels may simply be the result of sampling subjects for this experiment with eyelids relatively thinner than those sampled in the original study by Bierman et $\mathrm{al}^{19}$ based on which the light-dosing levels were determined. Another possibility is that, while asleep, subjects have much larger pupils than those used for the CS calculation, which was $2.3 \mathrm{~mm}$. Either or both of these effects would lead to much greater nocturnal melatonin suppression than expected from the CS calculation. Since it has now been demonstrated that light delivery can be successfully applied for both melatonin suppression and shifting circadian phase while subjects are asleep, it is important to explore these possibilities more systematically in the future.

In that context and with regard to practical applications, the present results have important clinical implications for future delivery of light while subjects are asleep. Certainly light delivery while sleeping would be much more convenient for people than, as is currently prescribed, requiring them to sit in front of a light box while awake for at least one hour. What makes this approach particularly appealing for clinical applications is that subjects were largely unaware of the light delivery while they slept in the present study. This observation is consistent with those from our previous study ${ }^{13}$ showing no meaningful differences in polysomnography recordings from subjects during light delivery while sleeping compared to sleeping without light delivery. While we did not measure polysomnograms in the present study, unpublished data from a pilot study using six subjects who had polysomnography recordings showed no significant difference in sleep efficiency after exposure to flashing blue light compared to no light exposure. From an engineering perspective, the demonstrated effectiveness of applying a train of blue light pulses through the eyelid during sleep makes the development of a sleep mask practical for home use because it avoids the significant buildup of heat from prolonged LED operation at intensities for closed eyelids. And from a safety perspective, the brief flashes of blue light minimize risk of blue light hazard, ${ }^{31}$ even if people might inadvertently have their eyelids open during the brief flashes. In the present study, subjects were advised to keep their eyes closed at all times while the light masks were energized to minimize discomfort glare from the bright light.

In conclusion, the present results furthered our basic understanding of circadian phototransduction and provide new insights into the development of new methods of delivering light to increase compliance and effectiveness of light treatment for circadian sleep and mood disorders.

\section{Acknowledgments}

This study was funded by Philips Respironics. The authors would like to thank Michael Colbaugh, Nathan Zimmerman, and Doug Mechlenburg from Philips Respironics for their support. The authors would also like to acknowledge 
Barbara Plitnick, Brittany Wood, Anna Lok, Robert Hamner, Sharon Lesage, Kenneth Appleman, Rebekah Mullaney, Ines Martinovic, and Dennis Guyon from the Lighting Research Center for their technical and editorial support.

\section{Disclosure}

The authors have jointly filed patent applications related to this work with Philips Respironics. The authors report no other conflicts of interest in this work.

\section{References}

1. Zeitzer JM, Ruby NF, Fisicaro RA, Heller HC. Response of the human circadian system to millisecond flashes of light. PLoS One. 2011;6(7): e22078.

2. Arvanitogiannis A, Amir S. Resetting the rat circadian clock by ultrashort light flashes. Neurosci Lett. 1999;261(3):159-162.

3. Vidal L, Morin LP. Absence of normal photic integration in the circadian visual system: response to millisecond light flashes. J Neurosci. 2007;27(13):3375-3382.

4. Kronauer RE, Forger DB, Jewett ME. Quantifying human circadian pacemaker response to brief, extended, and repeated light stimuli over the phototopic range. J Biol Rhythms. 1999;14(6):500-515.

5. Kronauer RE, Forger DB, Jewett ME. Erratum to: Quantifying human circadian pacemaker response to brief, extended, and repeated light stimuli over the photopic range. J Biol Rhythms. 2000;15(2):184-186.

6. Chang AM, Santhi N, St Hilaire M, et al. Human responses to bright light of different durations. J Physiol (Lond). 2012;590(Pt 13):3103-3112.

7. Wright $\mathrm{H}$, Lack LC. Effect of light wavelength on suppression and phase delay of the melatonin rhythm. Chronobiol Int. 2001;18(5):801-808.

8. Zeitzer JM, Dijk DJ, Kronauer R, Brown E, Czeisler C. Sensitivity of the human circadian pacemaker to nocturnal light: melatonin phase resetting and suppression. J Physiol (Lond). 2000;526(Pt 3):695-702

9. Figueiro MG, Lesniak NZ, Rea MS. Implications of controlled shortwavelength light exposure for sleep in older adults. BMC Res Notes. 2011;4:334.

10. Rea MS, Figueiro MG, Bullough JD, Bierman A. A model of phototransduction by the human circadian system. Brain Res Brain Res Rev. 2005;50(2):213-228.

11. Rea MS, Figueiro MG, Bierman A, Hamner R. Modeling the spectral sensitivity of the human circadian system. Light Res Tech. 2012;44(4): 386-396.

12. Figueiro MG, Rea MS, Bullough JD. Circadian effectiveness of two polychromatic lights in suppressing human nocturnal melatonin. Neurosci Lett. 2006(406):293-297.

13. Figueiro MG, Rea MS. Preliminary evidence that light through the eyelids can suppress melatonin and phase shift dim light melatonin onset. BMC Res Notes. 2012;5(1):221.

14. Berson DM, Dunn FA, Takao M. Phototransduction by retinal ganglion cells that set the circadian clock. Science. 2002;295(5557):1070-1073.
15. Belenky MA, Smeraski CA, Provencio I, Sollars PJ, Pickard GE. Melanopsin retinal ganglion cells receive bipolar and amacrine cell synapse. J Comp Neurol. 2003;460(3):380-393.

16. Hattar S, Lucas RJ, Mrosovsky N, et al. Melanopsin and rod-cone photoreceptive systems account for all major accessory visual functions in mice. Nature. 2003;424(6944):76-81.

17. Cao D, Zele AJ, Pokorny J. Linking impulse response functions to reaction time: rod and cone reaction time data and a computational model. Vision Res. 2007;47(8):1060-1074.

18. Baylor DA, Hodgkin AL, Lamb TD. The electrical response of turtle cones to flashes and steps of light. J Physiol (Lond). 1974;242(3): 685-727.

19. Bierman A, Figueiro MG, Rea MS. Measuring and predicting eyelid spectral transmittance. J Biomed Opt. 2011;16(6):067011.

20. Jewett ME, Rimmer DW, Duffy JF, Klerman EB, Kronauer RE, Czeisler CA. Human circadian pacemaker is sensitive to light throughout subjective day without evidence of transients. Am J Physiol. 1997;273(5 Pt 2):R1800-R1809.

21. Khalsa SB, Jewett ME, Cajochen C, Czeisler CA. A phase response curve to single bright light pulses in human subjects. J Physiol (Lond). 2003;549(Pt 3):945-952.

22. Roenneberg T, Wirz-Justice A, Merrow M. Life between clocks: daily temporal patterns of human chronotypes. J Biol Rhythms. 2003;18(1): $80-90$

23. Figueiro MG, Hamner R, Bierman A, Rea MS. Comparisons of three practical field devices used to measure personal light exposures and activity levels. Light Res Tech. 2013;45(4):421-434.

24. Rea MS, Figueiro MG, Bierman A, Bullough JD. Circadian light. J Circadian Rhythms. 2010;8(1):2.

25. Martin SK, Eastman CI. Sleep logs of young adults with self-selected sleep times predict the dim light melatonin onset. Chronobiol Int. 2002;19(4):695-707.

26. Voultsios A, Kennaway DJ, Dawson D. Salivary melatonin as a circadian phase marker: validation and comparison to plasma melatonin. J Biol Rhythms. 1997;12(5):457-466.

27. Smith MR, Revell VL, Eastman CI. Phase advancing the human circadian clock with blue-enriched polychromatic light. Sleep Med. 2009;10(3):287-294.

28. Molina TA, Burgess HJ. Calculating the dim light melatonin onset: the impact of threshold and sampling rate. Chronobiol Int. 2011;28(8): 714-718.

29. Czeisler CA, Duffy JF, Shanahan TL, et al. Stability, precision, and near-24-hour period of the human circadian pacemaker. Science. 1999;284(5423):2177-2181.

30. Illuminating Engineering Society of North America. IESNA Lighting Handbook: Reference and Application. 9th ed. Rea MS, editor. New York, NY: Illuminating Engineering Society of North America; 2000.

31. Bullough JD. The blue-light hazard: A review. J Illum Eng Soc. 2000;29(2):6-14.
Nature and Science of Sleep

\section{Publish your work in this journal}

Nature and Science of Sleep is an international, peer-reviewed, open access journal covering all aspects of sleep science and sleep medicine, including the neurophysiology and functions of sleep, the genetics of sleep, sleep and society, biological rhythms, dreaming, sleep disorders and therapy, and strategies to optimize healthy sleep. The journal welcomes

\section{Dovepress}

original research, clinical \& epidemiological studies, reviews \& evaluations, case reports and extended reports. The manuscript management system is completely online and includes a very quick and fair peerreview system, which is all easy to use. Visit http://www.dovepress.com/ testimonials.php to read real quotes from published authors. 